\title{
Management of Peritoneal Metastases Developed from Gastric Cancer: Laparascopic Hyperthermic Intraperitoneal Chemontherapy in Neoadjuvant Setting
}

Yutaka Yonemura $^{1 *}$, Emel Canbay ${ }^{1}$, Shouzou Sako ${ }^{1}$, Haruaki Ishibashi $^{2}$, Masamitu Hirano $^{3}$, Akiyoshi Mizumoto $^{3}$, Kazuyosi Takeshita $^{1}$, Kousuke Noguchi $^{3}$, Nobuyuki $\mathrm{TakaO}^{3}$, Masumi Ichinose ${ }^{3}$, Yang $\mathrm{Liu}^{4}$ and $\mathrm{Yan} \mathrm{Li}^{4}$

${ }^{1} \mathrm{NPO}$ Organization to Support Peritoneal Surface Malignancy Treatment, Osaka, Japan

${ }^{2}$ Peritoneal Dissemination Center, Kishiwada Tokushukai Hospital, Kishiwada, Japan

${ }^{3}$ Department of Surgery, Kusatsu General Hospital, Shiga, Japan

${ }^{4}$ Department of Oncology, Zhongnam Hospital, Cancer Center of Wuhan University, Wuhan, China

"Corresponding author: Yutaka Yonemura, Department of Regional Cancer Therapies, NPO to Support Peritoneal Disseminated Diseases, Kishiwada Tokushukai Hospital, 1-26, Haruki-Moto-Machi, Kishiwada City, Osaka, Japan, Tel, 81-090-9442-5173; E-mail: y.yonemura@coda.ocn.ne.jp

Rec date: Apr 24, 2014, Acc date: May 29, 2014, Pub date: June 6, 2014

Copyright: (c) 2014 Yonemura $\mathrm{Y}$, et al. This is an open-access article distributed under the terms of the Creative Commons Attribution License, which permits unrestricted use, distribution, and reproduction in any medium, provided the original author and source are credited.

\begin{abstract}
Objective: Peritoneal Metastases (PM) of Gastric Cancer (GC) are lesions of peritoneal surfaces, which may cause the dissemination throughout the abdominal cavity. The role of laparoscopic Hyperthermic Intraperitoneal Chemotherapy (HIPEC) as neoadjuvant purpose in the management of PM of GC is undefined.
\end{abstract}

Methods: Fifty patients were enrolled into this study with histopathological diagnosis of PM of GC referred to our center between 2012 and 2013 All patients were underwent two cycles of neoadjuvant laparoscopic HIPEC. At the second session of LHIPEC, ascites volume, cytological status and PCI levels were compared with those at the 1st LHIPEC.

Results: There was no intraoperative complication and mortality after LHIPEC. Four patients developed mild azotemia of Grade 2. Amount of ascites were completely abolished or decreased in 22 of $34(64.7 \%)$ and positive peritoneal cytology changed to be a negative in 14 of $20(70 \%)$ patients at the 2nd LHIPEC. Complete response was in $6(12 \%)$, and peritoneal cancer indices $(\mathrm{PCI})$ were significantly reduced from $14.3 \pm 10.2$ at the 1 st LHIPEC to $10.8 \pm 10.5$ at the 2 nd LHIPEC $(p<0.05)$. Furthermore, total $\mathrm{PCl}$ scores on small bowel mesentery at 1 st and 2 nd LHIPEC were $6.56 \pm 2.92$ and $5.25 \pm 3.78(P=0.016)$.

Conclusions: This study identified two outcomes. Diagnostic and therapeutic laparoscopy can be performed safely in patients with PM of GC. Laparoscopic HIPEC can be applied as a neoadjuvant treatment modality in order to reduce the tumor burden and disease control until complete managements to be achieved in patients with PM of GC.

Keywords: Laparoscopic chemohyperthermia; Gastric cancer; Peritoneal surface malignancy; Intraperitoneal chemotherapy.

\section{Introduction}

Peritoneal metastases (PM) of Gastric Cancer (GC) have been considered a terminal stage of disease [1]. The traditional goal of PM from GC has been palliation rather than curative. However, patients with PM do not respond to chemotherapy [2]. This occurs because systemic chemotherapies are penetrated less efficiently in peritoneal metastatic nodules of GC than disease at other sites. A poor response to systemic management provides the rationale for development of a intraperitoneal treatment of PM of GC.

Over the past three decades, a new multimodal treatment called Cytoreductive Surgery (CRS) with intraperitoneal chemotherapy (IPC) was proposed in management of PM of GC and yielded with prolonged survival [3-9]. The median survival time (MST) and 1 year survival of these patients was reported to be 6.5-11 months and 29.4-49.1\% respectively. Moreover, bidirectional intraperitoneal and systemic chemotherapy (BIPSC) was developed to reduce the tumor burden and to eradicate peritoneal free cancer cells prior to CRS and HIPEC in GC patients with PM [10]. This treatment was designed to eradicate dissemination from both peritoneum and subperitoneal blood vessels. Recently, this group published the results of treatment of 194 synchronous and metachronous GC patients with PM and the median survival rate was reached up to 15.8 months, with 1-, 2-, and 5 -year survival rates of 66,32 and $10.7 \%$, respectively [11].

Even if these treatments can be considered as effective in terms of survival, it is still limited. The use of laparascopy to perform HIPEC may be an interesting option because it permits exact evaluation of peritoneal cancer index (PCI) defined by Sugarbaker [12] and reduction of surgical trauma and faster recovery [13,14]. Laparascopic HIPEC was performed in pancreatic cancers and appendiceal mucinous neoplasms as neoadjuvant setting $[15,16]$. Here, we aimed to test hypothesis that laparascopic HIPEC (LHIPEC) can induce tumor regression and ascites control in GC patients with PM in neoadjuvant setting. This is the first report to clarify the reduction of PCI score after one session of LHIPEC. 
Citation: Yutaka Yonemura, Emel Canbay, Shouzou Sako, Haruaki Ishibashi, Masamitu Hirano Akiyoshi Mizumoto, Kazuyosi Takeshita, Kousuke Noguchi, Nobuyuki Takao, Masumi Ichinose, Yang Liu and Yan Li (2014) Management of Peritoneal Metastases Developed from Gastric Cancer: Laparascopic Hyperthermic Intraperitoneal Chemontherapy in Neoadjuvant Setting. J Integr Oncol 3: 117. doi: $10.4172 / 2329-6771.1000117$

Page 2 of 5

\section{Patients and Methods}

Neoadjuvant LHIPEC was performed in fifty GC patients with PM referred to Peritoneal Surface Malignacy Centre of Kusatsu general hospital between 2010 and 2013. Institutional review board approval was obtained at October, 26, 2012, as a title of "A study of the safety and efficacy of LHIPEC for the treatment of peritoneal metastasis from gastrointestinal cancer". All patients signed an informed consent form and underwent repeated 2 cycles of laparascopic HIPEC in neoadjuvant setting prior to cytoreductive surgery and hyperthermic intraperitoneal chemotherapy.

\section{Eligibility criteria}

The eligibility criteria included: (1) histologically or cytologically proven PM from GC; (2) absence of hematogenous metastasis and remote lymph node metastasis; (3) age 75 years or younger; (4) Eastern Clinical Oncology Group scale of performance status 3 or less; (5) good bone marrow, liver, cardiac, and renal function; (6) absence of severe adhesion in the peritoneal cavity; and (7) absence of other severe medical conditions or synchronous malignancy.

\section{Laproscopic HIPEC}

The patients were put under general anesthesia. A $12 \mathrm{~mm}$ blunt port was placed from the $2 \mathrm{~cm}$ longitudinal incision above the umbilicus. A second trocar $(12 \mathrm{~mm})$ was placed in the right Upper quadrant, following by a third trocar $(12 \mathrm{~mm})$ in the left lower quadrant. A $5 \mathrm{~mm}$ trocar was added if necessary in the left upper quadrant.

Ascites was suctioned and the amount was measured. Cytological examination was done using the aspirated ascites. If there is no ascites, peritoneal wash cytology was performed using aspirated saline recovered after intraperitoneal administration of $200 \mathrm{ml}$ of saline.

Biopsy specimens were routinely taken from the peritoneal nodules. Quantitative evaluation of PM in the entire abdominal cavity was done using the peritoneal carcinomatosis index (PCI) based on the regions involved in the abdominal cavity and the lesion size $[12,17]$.

Small bowel was divided into four sectors, (upper jejunum,, lower jejunum, upper ileum and lower ileum.). Total lesion size scores of the four sectors are expressed small bowel PCI (SB-PCI).

Adhesiolysis was done in patients with slight adhesion. However, adhesiolysis in patients with metachronous PM was not done because of the severe adhesion around the residual stomach and esophago jejunostomy due to the previous operation. In 23 patients, observation of sector 2 was not observed.

Following the confirmation of the diagnosis and PCI determination, a longitudinal $5 \mathrm{~cm}$ midline incision was made on the midline of the lower abdomen for open laparotomy. Three drainage tubes were placed on the bilateral subdiaphragmatic space for the inlet tubes and on the pelvic flexure for an outlet tube. Then, HIPEC was performed at 42 to 43 centigrade for 60 minutes adding 3 liter of saline plus 12.5 $\mathrm{mg} / \mathrm{m} 2$ of Mitomycin C with Cisplatin $(50 \mathrm{mg} / \mathrm{m} 2)$.

Second session of LHIPEC (2nd LHIPEC) was done one month after the first session of LHIPEC (1st LHIPEC) in all cases. At the 2nd LHIPEC, ascites volume, peritoneal cytological study and PCI were examined again.

\section{Edvaluation of complications}

Complications were graded according to the classification established by Dindo and colleagues [18].

\section{Statistical analyses}

All patients were followed and no patients were lost to follow-up. Outcome data were obtained from medical records and patients' interview. All statistical analyses were performed using SPSS software statistical computer package version 17 (SPSS Inc., Chicago, USA). The amount of ascites and PCI of the first and 2nd LHIPEC were analyzed by student T-test. Statistical significance was defined as a pvalue $\leq 0.05$.

\section{Results}

\section{Patients characteristics}

Male and female were 28 and 22, respectively; average age was 55.3 \pm 12.9 years old. Patients with synchronous and metachronous PM were 27 and 23, respectively and Clinicopathologic characteristics of GC patients with PM are given in Table 1

\begin{tabular}{|l|l|}
\hline male/female & $28 / 22$ \\
\hline primary/recurrence & $27 / 23$ \\
\hline mean age & $55.3 \pm 12.9$ \\
\hline histology & \\
\hline differentiated & 2 \\
\hline poorly differentiated & 48 \\
\hline LN metastasis & \\
\hline none & 9 \\
\hline positive & 51 \\
\hline macroscopic type & 15 \\
\hline type 3 & 35 \\
\hline type 4 & \\
\hline
\end{tabular}

Table 1: Patients characteristics

\section{Perioperative mortality and morbidity}

No intraoperative complication was experienced. There was no mortality after LHIPEC. In postoperative course, mild azotemia of Grade 2 was developed in four patients and was resolved until postoperative day 7. Mean hospital stay was 8.0 days (range 5-17).

\section{Effects on ascites and cytology}

The amounts of ascites at the 1st and 2nd LHIPEC were $731 \pm 1584$ and $334 \pm 752 \mathrm{ml}$, respectively. There was a significant difference between the two groups $(\mathrm{P}=0.039)$. Ascites was detected in 34 patients at 1st LHIPEC, and the amount of ascites decreased in 14 patients at the 2nd LHIPEC. In 8 of 34 patients, ascites was completely disappeared at the 2nd LHIPEC. However, seven patients showed increase of ascites. Ascites volume did not change in other 13 patients. 
Citation: Yutaka Yonemura, Emel Canbay, Shouzou Sako, Haruaki Ishibashi, Masamitu Hirano Akiyoshi Mizumoto, Kazuyosi Takeshita, Kousuke Noguchi, Nobuyuki Takao, Masumi Ichinose, Yang Liu and Yan Li (2014) Management of Peritoneal Metastases Developed from Gastric Cancer: Laparascopic Hyperthermic Intraperitoneal Chemontherapy in Neoadjuvant Setting. J Integr Oncol 3: 117. doi: 10.4172/2329-6771.1000117

Page 3 of 5

Cytology was positive in $20(40.0 \%)$ patients at the 1 st LHIPEC, and the positive cytology changed to be negative in 14 (70\%) of 20 patients at the 2nd LHIPEC.

\section{Changes of PCI levels at 1st and 2nd session of LHIPEC}

PCI at the 2nd session $(10.8 \pm 10.5)$ was significantly lower than that at the 1 st session $(14.3 \pm 10.2)(\mathrm{P}=0.05)$. PCI levels at the $2 \mathrm{nd}$ session changed to be 0 in 6 patients. Figure $1 \mathrm{~A}$ shows the laparoscopic findings of 1st LHIPEC on sector 1 in 73 years old man and the lesion size score on sector 1 has been 2 . At the 2 nd Laparoscopy, lesion size score was 0 (Figure 1B). In 25 patients, PCI levels at the 1st LHIPEC decreased at the 2 nd LHIPEC, and those of 20 patients were same at the 2nd LHIPEC. PCI levels of the 1st LHIPEC of the other 5 patients increased at the 2nd LHIPEC.

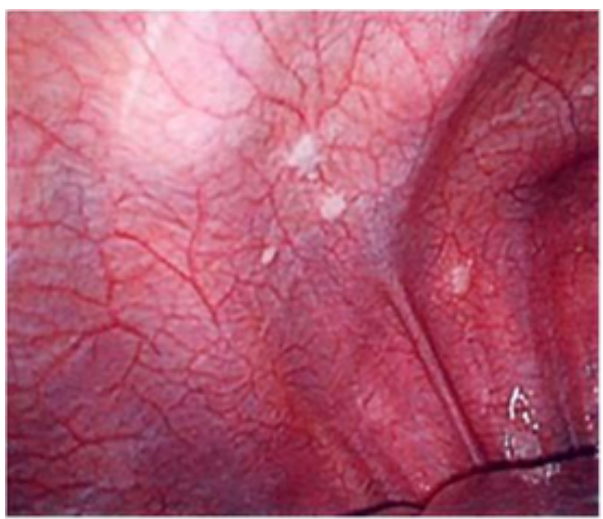

Figure 1A: Laparoscopic findings of sector 1 in 73 years old man at 1st LHIPEC. Lesion size score was 2

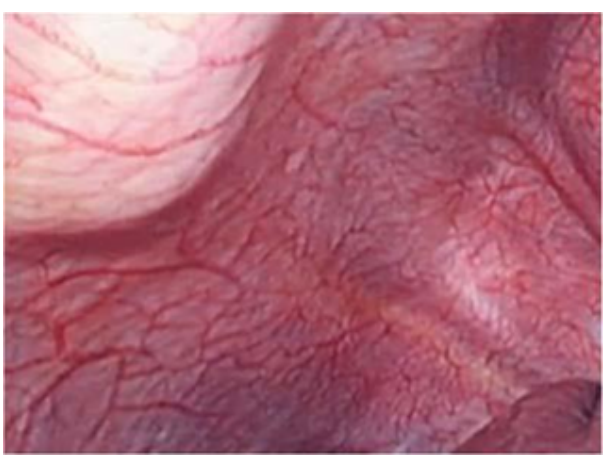

Figure 1B: Laparoscopic findings of sector 1 in 73 years old man at 2nd LHIPEC. Lesion size score was 0.

PCI levels of twenty-seven patients showed $\geq 11$. In 13 (48\%) of the 27 patients, PCI levels at 2 nd LHIPEC became to be $\leq 10$.

Table 2 showed the changes of the lesion size scores on each 13 peritoneal sectors. At second LHIPEC, the lesion size scores on right upper (sector 1), lower jejunum (sector 10), upper ileum (sector 11) and lower ileum (sector 12) were significantly lower than those at 1st LHIPEC. Furthermore, SB-PCIs (total lesion size scores on sector 9, 10,11 , and 12) at 1 st and 2nd LHIPEC were $6.56 \pm 2.92$ and $5.25 \pm$
3.78, respectively. There was a significant difference in SB-PCI levels between the two groups $(\mathrm{P}=0.016)$.

\begin{tabular}{|c|c|c|c|}
\hline \multirow[b]{2}{*}{ Sectors } & \multicolumn{2}{|c|}{ Lesion size (mean \pm S.D.) } & \multirow[b]{2}{*}{$\mathrm{p}$} \\
\hline & 1st LHIPEC & 2nd LHIPEC & \\
\hline 0 : central & $1.25 \pm 1.15$ & $1.06 \pm 1.12$ & NS \\
\hline 1: right upper & $1.57 \pm 1.09$ & $1.21 \pm 1.15$ & $P=0.027$ \\
\hline 2: epigastrium & $1.20 \pm 1.21$ & $1.42 \pm 0.99$ & NS \\
\hline 3: left upper & $1.33 \pm 1.23$ & $1.20 \pm 1.21$ & NS \\
\hline 4: left flank & $1.0 \pm 0.97$ & $0.88 \pm 0.96$ & NS \\
\hline 5: left lower & $1.25 \pm 1.29$ & $1.06 \pm 1.12$ & NS \\
\hline 6: pelvis & $1.52 \pm 1.26$ & $1.38 \pm 1.16$ & NS \\
\hline 7: right lower & $1.38 \pm 1.20$ & $1.25 \pm 1.18$ & NS \\
\hline 8: right flank & $1.02 \pm 1.03$ & $0.81 \pm 1.11$ & NS \\
\hline 9: upper jejunum & $1.44 \pm 0.89$ & $1.18 \pm 0.98$ & NS \\
\hline 10: lower jejunum & $1.56 \pm 0.81$ & $1.19 \pm 0.97$ & $P=0.014$ \\
\hline 11: upper ileu & $1.69 \pm 0.87$ & $1.38 \pm 1.02$ & $P=0.028$ \\
\hline 12: lower ileum & $1.88 \pm 0.72$ & $1.51 \pm 0.28$ & $P=0.014$ \\
\hline
\end{tabular}

Table 2: Lesion size scores in 13 sectors at 1st and 2nd LHIPEC

\section{Discussion}

The first report of cytoreductive surgery (CRS) and HIPEC in a patient with PC from gastric cancer dates back to 1988 [19]. Since then, CRS and HIPEC have been considered as the standard treatment for this group of patients even though there has been only one prospective randomized trial [8]. Analysis of the published data demonstrates that a complete surgical eradication is associated with the best outcome, and while the added benefit of the HIPEC to a complete cytoreduction has established in recent meta-analysis [20].

When it comes to the mode of the presentation of PM from gastric cancer, management has started to change with CRS plus HIPEC in the last 3 decades. The rationale of HIPEC is to treat the disease invaded to intraabdominal cavity and to control ascites.

The use of laparoscopy to perform HIPEC has shown to improve the effectiveness of intraperitoneal chemotherapy administration [22]. Prior to our study, LHIPEC in neoadjuvant setting was performed $[13,16,21]$. All the published series and our study report a very low morbidity rate and no deaths, and LHIPEC is considered as s very safe method. At the present time, LHIPEC as a neoadjyvant aim cannot be routinely recommended for wide application because of the lack of data on its real effectiveness.

This study clearly demonstrate the real effects of LHIPEC on PM from gastric cancer, such as decreased ascites, the eradication of peritoneal free cancer cells and decreased PCI levels. A remarkable effect in the present study was the decreased volume of ascites.

Malignant ascites is challenging clinical problem in cancer patients, which have a major impact on quality of life. The prognosis of malignant ascites is poor with a median survival of only 5.7 months 
Citation: Yutaka Yonemura, Emel Canbay, Shouzou Sako, Haruaki Ishibashi, Masamitu Hirano Akiyoshi Mizumoto, Kazuyosi Takeshita, Kousuke Noguchi, Nobuyuki Takao, Masumi Ichinose, Yang Liu and Yan Li (2014) Management of Peritoneal Metastases Developed from Gastric Cancer: Laparascopic Hyperthermic Intraperitoneal Chemontherapy in Neoadjuvant Setting. J Integr Oncol 3: 117. doi: 10.4172/2329-6771.1000117

Page 4 of 5

[23]. Although paracentesis, diuretics and shunting are commonly used procedures as treatment of malignant ascites, the efficacy and the results are often temporary.

In the present study, ascites was decreased in $14(41 \%)$ of 34 patients, and was completely disappeared in $8(24 \%)$ of 14 patients after one session of LHIPEC. The complete clinical regression of ascites was found in all the 58 patients, who have been treated by LHIPEC [20].

Ascites is developed by the vasoactive cytokines, produced from intraperitoneal inflammatory cells and cancer cells. Vascular endothelial growth factor (VEGF) is a well-known cytokine, which increase the vascular permeability. VEGF mRNA is expressed from all the gastric cancer cell lines and clinical samples [23]. VEGF produced from cancer cells increases permeability of submesothelial vasculature, and plays an important role in ascites development in gastric cancer [24]. VEGF levels in ascites of patients with high PCI were significantly higher than those with low PCI [24]. After wash out the ascites and peritoneal free cancer cells by LHIPEC, the intraperitoneal VEGF levels may be decreased. As a result, the pearmeability of submesothelial vasclature may be decreased, resulting in the regression of ascites.

Systemic chemotherapies have minimal effects on PM [25]. The peritoneal cavity acts as a sanctuary against systemic chemotherapy, probably because of the existence of a blood-peritoneal barrier consisting of stromal tissue between mesothelial cells and submesothelial blood capillaries [26]. This barrier acrosses for a total thickness of $90 \mu \mathrm{m}$ [26]. Accordingly, only a small amount of systemic drugs are capable of penetrating this barrier and passing into the peritoneal cavity. Accordingly, systemic chemotherapy cannot efficiently eradicate peritoneal free cancer cells and established PM [27]. In contrast, peritoneal free cancer cells could be efficiently treated by the intraperitoneal chemotherapy, because high loco-regional intensity can be obtained by the intarperitoneal chemotherapy.

Yonemura et al. reported that the positive cytology results became negative in $69 \%(47 / 68)$ of patients with PM from gastric cancer after six cycles of intraperitoneal administration of docetaxel and cisplatin [28]. The present study demonstrated that the positive cytology at the 2nd LHIPEC changed to be negative in $14(70 \%)$ of 20 patients after one session of LHIPEC. Positive peritoneal cytology is a sign of poor prognosis even if there is no macroscopic PM [6]. In contrast, the prognosis of patients with macroscopic PM and negative cytologic study had significant better survival than those with PM and positive cytology [6]. Accordingly, LHIPEC is a useful neoadjuvant therapy for the eradication of peritoneal free cancer cells before CRS. In addition, cytological study at 2nd laparoscopy has a role in the selection of patients for the CRS or further chemotherapy. If the cytology at the 2nd LHIPEC remains positive results, patients must be treated further chemotherapy.

The most important finding of the present study is that LHIPEC decreased the PCI levels. So far, there have been no reports about the reduction of tumor burden after LHIPEC. The present study demonstrated that PCI at the 2nd LHIPEC was significantly lower than that at the 1st LHIPEC. In 25 patients, PCI levels at the 1 st LHIPEC decreased at the 2nd LHIPEC, Furthermore, PCI levels at the 2nd LHIPEC changed to be 0 in 6 patients, and the complete response rate was $12 \%$.

At the 1st staging laparoscopy, almost one-half of the patients were excluded from the candidates for CRS, because the PCI was higher than the threshold level [13]. Accordingly, many of the patients are submitted to a second staging laparoscopy after systemic neoadjuvant chemotherapy. Glehen [6] and Yonemura (29) reported that patients with PCI level $\leq 10$ survived significantly better than those with PCI $\geq$ 11 . In the present study, PCI levels of twenty-seven patients showed $\geq$ 11 at the 1st LHIPEC. In 13 (48\%) of the 27 patients, PCI levels at 2nd LHIPEC became to be $\leq 10$.

Accordingly, LHIPEC can decrease the PCI levels under the threshold level corresponding with good prognosis, and 2nd laparoscopy can serve as a selection criterion for an evaluation tool of optimal candidates for cytoreductive surgery [29].

After CRS for PM from gastric cancer, the complete cytoreduction is the strongest independent prognostic factor. However, diffuse small bowel involvement is the most frequent cause of the incomplete cytoreduction.

Valle reported that CC- 0 can be achieved only in fewer than $30 \%$ of cases with involvement in sectors from 9 to 12 [13]. The degree of involvement of the small bowel turns out to be the true cutoff point about chance to achieve CC- 0 resection. The present study showed that lesion size scores at the 2nd LHIPEC on the small bowel and right diaphragmatic peritoneum significantly decreased after 1st LHIPEC. Accordingly, LHIPEC can reduce the SB-PCI, resulting in the improvement of complete cytoreduction rate and the preservation of intact small bowel.

In conclusions, this new method is not only a safe method, but also an effective treatment to control malignant ascites and to eradicate peritoneal free cancer cells before CRS. Hyperthermia higher than 41 centigrade is known to increase the drug penetration distance from the peritoneal surface [30]. Laparoscopic HIPEC allows for the better penetration of drugs in peritoneal tumors than HIPEC under laparotomy, because closed HIPEC generates a higher intraperitoneal pressure than HIPEC under laparotomy [31].

Furthermore, LHIPEC can reduce PCI and SB-PCI levels, resulting in the increase of complete cytoreduction rates. Further large prospective studies are necessary for determination of laparoscopy as a potential standard of a diagnostic and therapeutic algorithm in patients with PM from GC.

\section{References}

1. Sadeghi B, Arvieux C, Glehen O, Beaujard AC, Rivoire M, et al. (2000) Peritoneal carcinomatosis from non-gynecologic malignancies: results of the EVOCAPE 1 multicentric prospective study. Cancer 88: 358-363.

2. Ajani JA, Ota DM, Jessup JM, Ames FC, McBride C, et al. (1991) Resectable gastric carcinoma. An evaluation of preoperative and postoperative chemotherapy. Cancer 68: 1501-1506.

3. Fujimoto S, Takahashi M, Mutou T, Kobayashi K, Toyosawa T, et al. (1997) Improved mortality rate of gastric carcinoma patients with peritoneal carcinomatosis treated with intraperitoneal hyperthermic chemoperfusion combined with surgery. Cancer 79: 884-891.

4. Hall JJ, Loggie BW, Shen P, Beamer S, Douglas Case L, et al. (2004) Cytoreductive surgery with intraperitoneal hyperthermic chemotherapy for advanced gastric cancer. J Gastrointest Surg 8: 454-463.

5. Yonemura Y, Kawamura T, Bandou E, Takahashi S, Sawa T, et al. (2005) Treatment of peritoneal dissemination from gastric cancer by peritonectomy and chemohyperthermic peritoneal perfusion. Br J Surg 92: 370-375.

6. Glehen O, Gilly FN, Arvieux C, Cotte E, Boutitie F, et al. (2010) Peritoneal carcinomatosis from gastric cancer: a multi-institutional study of 159 patients treated by cytoreductive surgery combined with 
Citation: Yutaka Yonemura, Emel Canbay, Shouzou Sako, Haruaki Ishibashi, Masamitu Hirano Akiyoshi Mizumoto, Kazuyosi Takeshita, Kousuke Noguchi, Nobuyuki Takao, Masumi Ichinose, Yang Liu and Yan Li (2014) Management of Peritoneal Metastases Developed from Gastric Cancer: Laparascopic Hyperthermic Intraperitoneal Chemontherapy in Neoadjuvant Setting. J Integr Oncol 3: 117. doi: $10.4172 / 2329-6771.1000117$

Page 5 of 5

perioperative intraperitoneal chemotherapy. Ann Surg Oncol 17: 2370-2377.

7. Ströhlein MA, Bulian DR, Heiss MM (2011) Clinical efficacy of cytoreductive surgery and hyperthermic chemotherapy in peritoneal carcinomatosis from gastric cancer. Expert Rev Anticancer Ther 11: 1505-1508.

8. Yang XJ, Huang CQ, Suo T, Mei LJ, Yang GL, et al. (2011) Cytoreductive surgery and hyperthermic intraperitoneal chemotherapy improves survival of patients with peritoneal carcinomatosis from gastric cancer: final results of a phase III randomized clinical trial. Ann Surg Oncol 18: 1575-1581.

9. Magge D, Zenati M, Mavanur A, Winer J, Ramalingam L, et al. (2014) Aggressive locoregional surgical therapy for gastric peritoneal carcinomatosis. Ann Surg Oncol 21: 1448-1455.

10. Yonemura Y, Bandou E, Sawa T, Yoshimitsu Y, Endou Y, et al. (2006) Neoadjuvant treatment of gastric cancer with peritoneal dissemination. Eur J Surg Oncol 32: 661-665.

11. Canbay E, Mizumoto A, Ichinose M, Ishibashi H, Sako S, et al. (2013) Outcome data of patients with peritoneal carcinomatosis from gastric origin treated by a strategy of bidirectional chemotherapy prior to cytoreductive surgery and hyperthermic intraperitoneal chemotherapy in a single specialized center in Japan. Ann Surg Oncol 21: 1147-1152.

12. Harmon RL, Sugarbaker PH (2005) Prognostic indicators in peritoneal carcinomatosis from gastrointestinal cancer. Int Semin Surg Oncol 2: 3 .

13. Valle M, Federici O, Garofalo A (2012) Patient selection for cytoreductive surgery and hyperthermic intraperitoneal chemotherapy, and role of laparoscopy in diagnosis, staging, and treatment. Surg Oncol Clin N Am 21: 515-531.

14. Sommariva A, Zagonel V, Rossi CR (2012) The role of laparoscopy in peritoneal surface malignancies selected for hyperthermic intraperitoneal chemotherapy (HIPEC). Ann Surg Oncol 19: 3737-3744.

15. Chang E, Alexander HR, Libutti SK, Hurst R, Zhai S, et al. (2001) Laparoscopic continuous hyperthermic peritoneal perfusion. J Am Coll Surg 193: 225-229.

16. Hirano M, Yonemura Y, Canbay E, Ichinose M, Togawa T, et al. (2012) Laparascopic diagnosis and laparascopic hypothermic intraoperative intraperitoneal chemotherapy for pseudomyxoma peritonei detected by CT examination. Gastroenterol Pract 2012: 741202.

17. Sugarbaker PH (2007) Cytoreduction surgery and perioperative intraperitoneal chemotherapy as a curative approach to pseudomyxoma syndrome. Eur J Surg Oncol 27: 239-243.

18. Dindo D, Demartines N, Clavien PA (2004) Classification of surgical complications: a new proposal with evaluation in a cohort of 6336 patients and results of a survey. Ann Surg 240: 205-213.

19. Ayantunde AA, Parsons SL (2007) Pattern and prognostic factors in patients with malignant ascites: a retrospective study. Ann Oncol 18: 945-949.
20. Coccolini F, Cotte E2, Glehen O2, Lotti M3, Poiasina E3, et al. (2014) Intraperitoneal chemotherapy in advanced gastric cancer. Meta-analysis of randomized trials. Eur J Surg Oncol 40: 12-26.

21. Esquivel J (2007) Cytoreductive surgery for peritoneal malignancies-development of standards of care for the community. Surg Oncol Clin N Am 16: 653-666, $\mathrm{x}$

22. Valle M, Van der Speeten K, Garofalo A (2009) Laparoscopic hyperthermic intraperitoneal preoperative chemotherapy (HIPEC) in the management of refractory malignant ascites: A multi-institutional retrospective analysis in 52 patients. J Surg Oncol 15: 331-334.

23. Yonemura Y, Endo Y, Tabata K, Kawamura T, Yun HY, et al. (2005) Role of VEGF-C and VEGF-D in lymphangiogenesis in gastric cancer. Int J Clin Oncol 10: 318-327.

24. Fushida S, Oyama K, Kinoshita J, Yagi Y, Okamoto K, et al. (2013) VEGF is a target molecule for peritoneal metastasis and malignant ascites in gastric cancer: prognostic significance of VEGF in ascites and efficacy of anti-VEGF monoclonal antibody. Onco Targets Ther 6: 1445-1451.

25. Markman M (1991) Intraperitoneal therapy in ovarian cancer utilizing agents acjieving high local but low systemic exposure. Reg Cancer Treat 0: 256-60.

26. Baron MA (1941) Structure of intestinal peritoneum in man. Am J Anat 69: 439-497.

27. Sugarbaker PH, Mora JT, Carmignani P, Stuart OA, Yoo D (2005) Update on chemotherapeutic agents utilized for perioperative intraperitoneal chemotherapy. Oncologist 10: 112-122.

28. Yonemura Y (2012) Effects of Neoadjuvant Intraperitoneal/Systemic Chemotherapy (Bidirectional Chemotherapy) for the Treatment of Patients with Peritoneal Metastasis from Gastric Cancer. International J Surg Oncol vol.

29. Yonemura Y, Elnemr A, Endou Y, Ishibashi H, Mizumoto A, Miura M, Yan LI. (2012) Surgical Results of Patients with Peritoneal Carcinomatosis Treated with Cytoreductive Surgery Using a New Technique Named Aqua Dissection. Gastroenterol Res Pract 2012: 521487.

30. Los G, Mutsaers PH, van der Vijgh WJ, Baldew GS, de Graaf PW, et al. (1989) Direct diffusion of cis-diamminedichloroplatinum(II) in intraperitoneal rat tumors after intraperitoneal chemotherapy: a comparison with systemic chemotherapy. Cancer Res 49: 3380-3384.

31. Thomas F, Ferron G, Gesson-Paute A, Hristova M, Lochon I, et al. (2008) Increased tissue diffusion of oxaliplatin during laparoscopically assisted versus open heated intraoperative intraperitoneal chemotherapy. Ann Surg Oncol 15: 3623-3624. 